\title{
Localization of Low Lying Eigenmodes for Chirally Symmetric Dirac Operator
}

\section{M.I. Polikarpov*, F.V. Gubarev, S.M. Morozov and}

ITEP, B. Cheremushkinskaya 25, Moscow, 117259 Russia

E-mail: polykarp@itep.ru, gubarev@itep.ru, smoroz@itep.ru

\section{V.I. Zakharov}

MPI, Föhringer Ring 6, 80805, München, Germany

E-mail: xxz@mppmu.mpg.de

We consider properties of zero and near-zero modes for overlap fermion operator in SU(2) lattice gluodynamics. The density of the states is of the order of $\Lambda_{Q C D}$ while the localization volume of the modes tends to zero in physical units with the lattice spacing tending to zero. The situation changes drastically when we study "vortex removed" configurations.

XXIIIrd International Symposium on Lattice Field Theory

25-30 July 2005

Trinity College, Dublin, Ireland

${ }^{*}$ Speaker. 


\section{Introduction}

The material of this talk has a substantial overlap with that presented in the talks of N. Cundy, C. Gattringer, T. De Grand, J. Greensite, J. Hetrick, I. Horvath, Y. Koma, S. Solbrig, B. Svetitsky, S. Syritsyn.

We study SU(2) lattice gluodynamics and in our calculations we use the massless overlap Dirac operator [1]:

$$
D=\frac{\rho}{a}\left(1+\frac{A}{\sqrt{A A^{\dagger}}}\right), \quad A=D_{W}-\frac{\rho}{a},
$$

where $A$ is the Wilson Dirac operator with negative mass term. Anti-periodic (periodic) boundary conditions in time (space) directions were employed. It turns out that for SU(2) gluodynamics the optimal value of $\rho$ parameter is 1.4. Furthermore, we have used the minmax polynomial approximation [2] to compute the sign function $\operatorname{sign}(A)=A / \sqrt{A A^{\dagger}} \equiv \gamma_{5} \operatorname{sign}(H)$, where $H=\gamma_{5} A$ is hermitian Wilson Dirac operator. In order to improve the accuracy and performance about one hundred lowest eigenmodes of $H$ were projected out. Note that the eigenvalues of (1.1) lie on the circle of radius $\rho$ centered at $(\rho, 0)$ in the complex plane. In order to relate them with continuous eigenvalues of the Dirac operator the circle was stereographically projected onto the imaginary axis [3]. The information about statistics and number of gauge field configurations used can be found in [4].

Below we discuss localization properties of eigenmodes of the overlap Dirac operator. A natural measure of the localization is provided by the inverse participation ratio (IPR) $I_{\lambda}$ which is defined as follows (for review see for example Ref. [5]). Let

$$
\rho_{\lambda}(x)=\psi_{\lambda}^{\dagger}(x) \psi_{\lambda}(x), \quad \sum_{x} \rho_{\lambda}(x)=1
$$

where $\psi_{\lambda}(x)$ is an eigenmode of the overlap Dirac operator in a given gauge field background with virtuality $\lambda, D \psi_{\lambda}=\lambda \psi_{\lambda}$. Then for any finite volume $V$ the IPR $I_{\lambda}$ is defined by

$$
I_{\lambda}=V \sum_{x} \rho_{\lambda}^{2}(x)
$$

and characterizes the inverse fraction of sites contributing to the support of $\rho_{\lambda}(x)$. Note that for delocalized modes $\rho_{\lambda}(x)=1 / V$ and hence $I_{\lambda}=1$, while an extremely localized mode, $\rho_{\lambda}(x)=$ $\delta_{x, x_{0}}$, is characterized by $I_{\lambda}=V$. Moreover, for eigenmodes localized on a fraction $f$ of sites (so that $\sup \rho_{\lambda}=V_{f}=f V$ ) we have $I_{\lambda}=V / V_{f}=1 / f$.

\section{Dependence of the IPR on the eigenvalue}

In Fig. 1 we show IPR for various lattice spacings at fixed physical volume. Two different effects are clearly observed. First, the IPR grows as function of $\lambda_{n}$ with $\lambda_{n} \rightarrow 0$, for fixed total volume and fixed lattice spacing. Second, for sufficiently small, fixed values of $\lambda_{n}$ the IPR grows with the lattice spacing tending to zero. An alternative way of representing the growth of IPR with $a \rightarrow 0$ is provided by the data on Fig. 2. Here we plot the ratio, $R$ of the densities of the eigenmodes with IPR $>5$ and with IPR $<5$ for two values of the lattice spacing $a$. The data demonstrate that 


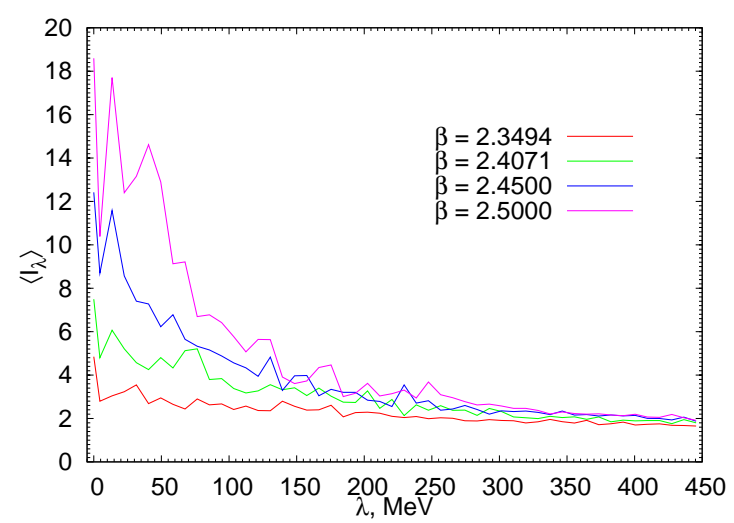

Figure 1: IPRs for low lying eigenmodes at various lattice spacings and fixed physical volume. The "mobility edge" $\lambda_{c r} \approx 150-200 \mathrm{MeV}$ is clearly seen.

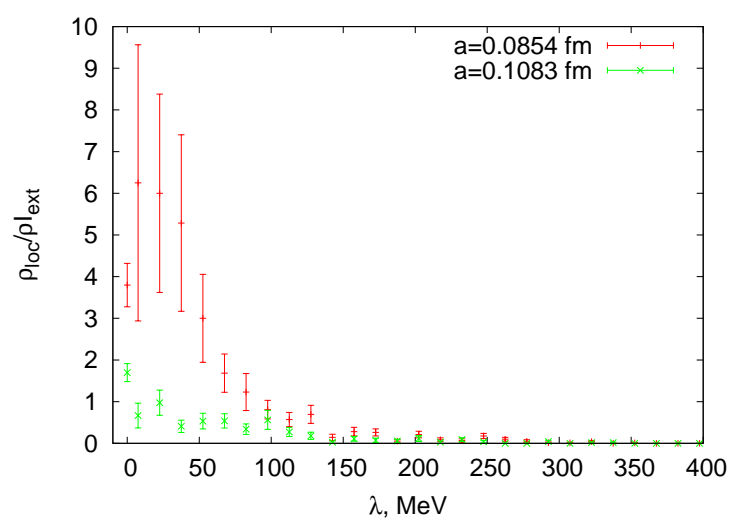

Figure 2: The ratio of the densities of the eigenmodes with IPR $>5$ to that with $\mathrm{IPR}<5$.

the observed growth of the IPR with $a \rightarrow 0$ is due to 'typical' eigenmodes, not due to a few modes with a huge value of the IPR.

Another qualitative feature of the data is existence of a kind of a 'merging point', $\lambda_{\text {merge }} \approx$ $150-200 \mathrm{MeV}$. Namely, for $\lambda_{n}>\lambda_{\text {merge }}$ the values of IPR practically do not depend on the eigenvalue $\lambda_{n}$ and the lattice spacing $a$. Note that existence of a point where all the curves giving the dependence of the IPR on $\lambda_{n}$ is also seen in the data on localization properties of eigenfunctions of a test color scalar particle [6]. Our data indicate scaling in physical units, at least an approximate one, of the position of the merging point.

Thus, our data on the localization properties of the low-lying eigenfunctions of the Dirac operator indicate existence of a variety of phenomena. If we look for analogies into solid-state physics (reviewed in, e.g., [5]) then, probably, one can find an analog to the growth of the IPR as function of $\lambda_{n}$ with diminishing $\lambda_{n}$. Indeed, it is known that if one approaches the mobility edge, $\lambda_{c r}{ }^{1}$ from above, then the IPR blows up as $\left(\lambda_{n}-\lambda_{c r}\right)^{-\alpha}$ where $\alpha>0$. Note that this

\footnotetext{
${ }^{1}$ Let us remind the reader that the mobility edge is defined in terms of dependence of the IPR on the total volume of the system, $V_{t o t}$. Namely, (IPR) $\sim V_{\text {tot }}$ for $V_{\text {tot }} \rightarrow \infty$ below the mobility edge, $\lambda_{n}<\lambda_{c r}$ and $(I P R) \sim$ const for $V_{\text {tot }} \rightarrow \infty$ above the mobility edge, $\lambda_{n}<\lambda_{c r}$. Our data on the dependence of the IPR on the total volume can be found in the original paper [4].
} 


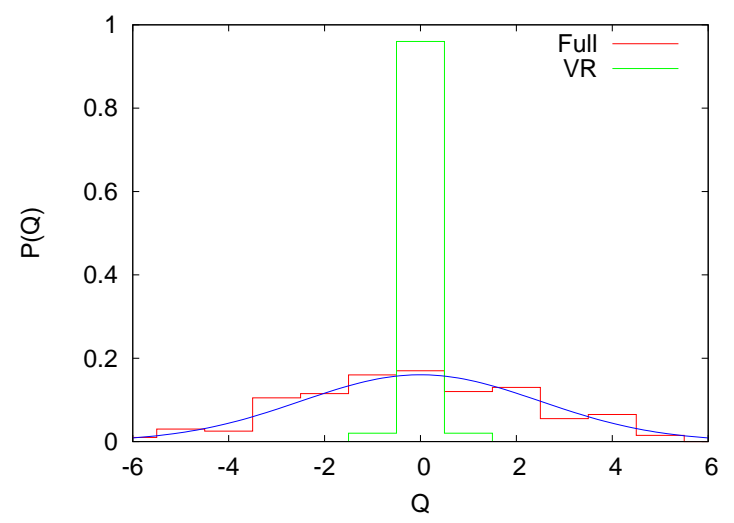

Figure 3: The histogram of the distribution of the topological charge for full and vortex removed (VR) configurations.

simple dependence holds for a fixed, large total volume $V_{t o t}$ and the singularity is smoothened due to finiteness of the total volume. Now, for chiral fermions one expects that the mobility edge is $\lambda_{c r}=0$. Then, the growth of the IPR with $\lambda_{n} \rightarrow 0$ indicated by our data (see Fig. 1) is reminiscent of existence of the critical exponent $\alpha$ in the non-relativistic case.

Since the data exhibit strong dependencies of the IPR on various variables, detailed analysis of these dependencies goes beyond the scope of the present study (some details, though, can be found in the original paper [4]). Here, we confine ourselves mostly to discussion of qualitative effects. From this point of view dependence on the ultraviolet cut off, that is the lattice spacing $a$, seems to be most exciting. We will discuss this point further in Sect. 4.

\section{Removing of central vortices}

The vortex-removing procedure [7] drastically changes the properties of the vacuum: confinement and chiral condensate disappear. Below we describe how the vortex removing acts on topological susceptibility of the vacuum, distribution of the Dirac operator eigenvalues and localization properties of the eigenmodes. On Fig.3 we show the distribution of the topological charge for full and vortex-removed configurations. It occurs that topological susceptibility, $\left\langle Q^{2} / V\right\rangle$, vanishes after removing of the center vortices. The topological charge of the configuration was defined by the index of overlap Dirac operator $Q=n_{+}-n_{-}$and $n_{ \pm}$is the number of exact zero modes with positive (negative) chirality.

On Fig.4 (left) we show the quantity $<\pi \rho(\lambda)>^{\frac{1}{3}}$ which is related to chiral condensate through the Banks-Casher relation [8], $\langle\bar{\psi} \psi\rangle=-\pi \rho\left(\lambda_{n} \rightarrow 0\right)$, where $\rho\left(\lambda_{n} \rightarrow 0\right)$ is the density of the (delocalized) zero modes in the limit of infinite volume $V$. For finite lattice volumes these modes are near-zero and this is the reason of the limit $\lambda_{n} \rightarrow 0$. It is seen that for the vortex removed configurations there exist the gap in the distribution of the near-zero eigenvalues, thus chiral condensate vanishes for vortex removed gauge fields.

On Fig.4 (right) IPR for full and vortex removed configurations is shown. After vortex removing all modes (except of a few surviving zero modes) become delocalized, $(I P R) \approx 1$. 

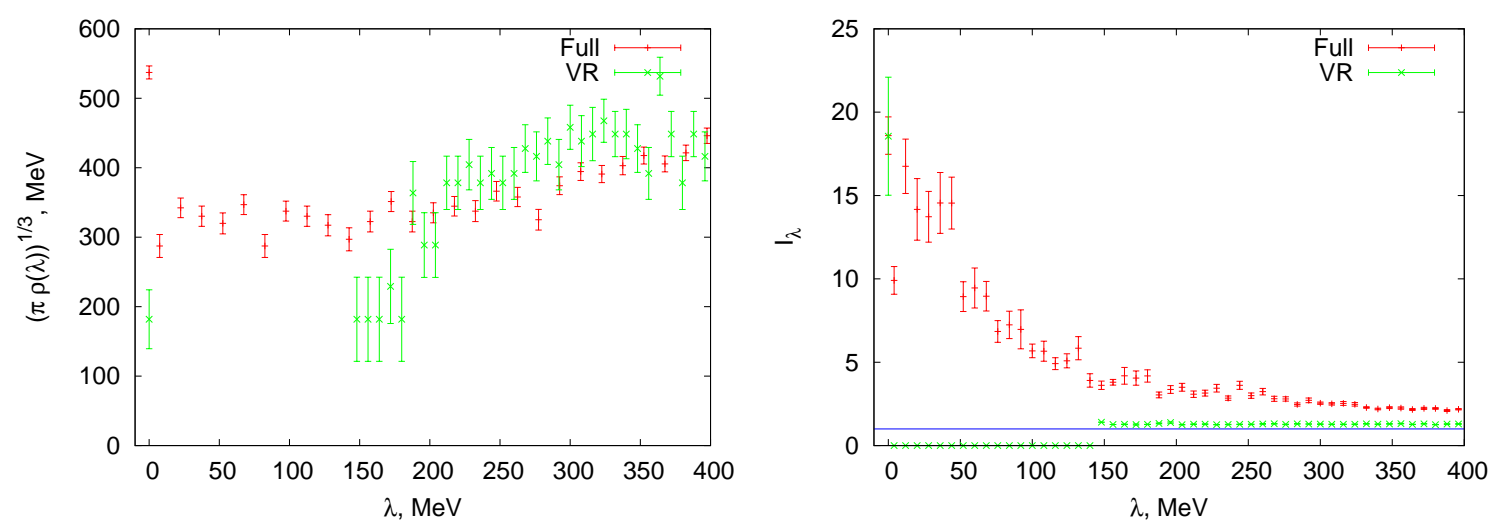

Figure 4: $\left\langle\pi \rho(\lambda)>^{\frac{1}{3}}\right.$ (left) and IPR (right) for full and vortex removed (VR) configurations. The average is taken over 200 gauge field configurations at $\beta=2.5$ on lattice $16^{3} \cdot 18$.

Thus, we can conclude that the non-trivial localization properties of the eigenfunctions of the Dirac operator are directly related to the central vortices. Note that similar observations were made by using other types of lattice fermions, see in particular [9] and references therein.

\section{Discussions}

The most striking feature exhibited by the data is a strong dependence of the IPR on the lattice spacing $a$. The data were fitted [4] by

$$
I_{\lambda}=b_{0}+b_{1} \cdot a^{d-4}
$$

where $b_{0,1}, d$ are constants to be determined from the fit. The IPR data for the low lying modes strongly suggest that

$$
d \approx 0
$$

In other words, the volume occupied by the eigenfunctions tends to zero in physical units as $a^{4}$.

Note that the $a$ dependence of the IPR was suggested first by the data of Ref. [10]. The latticespacing dependence exhibited by our data is considerably stronger than that reported in Ref. [10]. In particular, in Ref. [10] the values of IPR were changing, say, between $(I P R) \approx 2$ and $(I P R) \approx 3$ as a result of varying the lattice spacing. In our case, the highest values of IPR are approaching the value of 20. At this moment, it is difficult to clarify the reason for this difference. First, we use different types of fermions. Also, the color group is different, as well as the lattice action used.

The strong $a$ dependence of the IPR is in sharp contrast with scaling, in physical units, of the density of states and of the chiral condensate (defined through the Casher-Banks relation). Thus, the emerging picture is that the size of the eigenfunctions shrinks strongly with $a \rightarrow 0$ while the eigenvalues remain stable. In other words, the data suggest that the phenomenon of fine tuning is quite a common feature of the Yang-Mills dynamics ${ }^{2}$.

\footnotetext{
${ }^{2}$ The standard definition of the fine tuning, which goes back to the Higgs physics, is as follows. If a relativistic system has a typical size $r_{0}$ then the energy levels of order $\lambda \sim 1 / r_{0}$ are 'natural'. If, on the other hand, $\lambda \ll 1 / r_{0}$ the level is 'fine tuned'.
} 
Note that the phenomenon of the fine tuning was observed first by studying the properties of the lattice monopoles and vortices, for a review see, e.g., [11]. However the definition of the monopoles and vortices involves projected fields which makes theoretical interpretation more difficult. The fine tuning seen in the data on the fermion localization is defined in explicitly gauge invariant terms.

\section{Acknowledgments}

The invaluable assistance of G. Schierholz and T. Streuer in the overlap operator implementation is kindly acknowledged. This work was partially supported by grants RFBR-05-02-16306a, RFBR-05-02-17642, RFBR-0402-16079, RFFI-05-02-17642, RFBR-03-02-16941 and EU Integrated Infrastructure Initiative Hadron Physics (I3HP) under contract RII3-CT-2004-506078. F.V.G. was partially supported by INTAS YS grant 04-83-3943.

\section{References}

[1] H. Neuberger, Exactly massless quarks on the lattice, Phys. Lett. B 417 (1998) 141 [arXiv: hep-lat/9707022], More about exactly massless quarks on the lattice, Phys. Lett. B 427 (1998) 353 [hep-lat/9801031].

[2] L. Giusti, C. Hoelbling, M. Luscher and H. Wittig, Numerical techniques for lattice QCD in the epsilon regime, Comput. Phys. Commun. 15331 (2003) [hep-lat/0212012].

[3] S. Capitani, M. Gockeler, R. Horsley, P. E. L. Rakow and G. Schierholz, Operator improvement for Ginsparg-Wilson fermions, Phys. Lett. B 468 (1999) 150 [hep-lat/9908029].

[4] F.V. Gubarev, S.M. Morozov, M.I. Polikarpov and V.I. Zakharov, Low lying eigenmodes localization for chirally symmetric Dirac operator, preprint ITEP-LAT-2005-08, [hep-lat/0505016].

[5] B. Kramer and A. MacKinnon, Localization: theory and experiment, Rep.Prog.Phys. 56 (1993) 1469.

[6] J. Greensite, S. Olejnik, M.I. Polikarpov, S.N. Syritsyn and V.I. Zakharov, Localized eigenmodes of covariant laplacians in the Yang-Mills vacuum, Phys. Rev. D71 (2005) 114507 [hep-lat/0504008].

[7] P. de Forcrand, M. D'Elia, On the relevance of center vortices to QCD, Phys. Rev. Lett. 82 (1999) 4582 [hep-lat/9901020].

[8] T. Banks and A. Casher, Chiral symmetry breaking in confining theories, Nucl. Phys. B169 (1980) 103.

[9] J. Gattnar et al., Center vortices and Dirac eigenmodes in su(2) lattice gauge theory, Nucl. Phys. B716 (2005) 105.

[10] C. Aubin et al., [MILC Collaboration], The scaling dimension of low lying Dirac eigenmodes and of the topological charge density, [hep-lat/0410024].

[11] V. I. Zakharov, Lattice SU(2) theory projected on scalar particles, Phys. Usp. 47 (2004) 37, Usp. Fiz. Nauk 47 (2004) 39; Nonperturbative match of ultraviolet renormalon, [hep-ph/0309178]. 